University of Wollongong

Research Online

Faculty of Engineering and Information

Faculty of Engineering and Information

Sciences - Papers: Part A

Sciences

$1-1-2014$

\title{
Mitigating tap-changer limit cycles in modern electricity networks embedded with local generation units
}

Dothinka Ranamuka Rallage

University of Wollongong, dssrr987@uowmail.edu.au

Ashish Agalgaonkar

University of Wollongong, ashish@uow.edu.au

Kashem M. Muttaqi

University of Wollongong, kashem@uow.edu.au

Md Jan E Alam

University of Wollongong, mjealam@uowmail.edu.au

Follow this and additional works at: https://ro.uow.edu.au/eispapers

Part of the Engineering Commons, and the Science and Technology Studies Commons

Research Online is the open access institutional repository for the University of Wollongong. For further information contact the UOW Library: research-pubs@uow.edu.au 


\title{
Mitigating tap-changer limit cycles in modern electricity networks embedded with local generation units
}

\author{
Abstract \\ Cascaded on-load tap changers (OLTC) are widely used for coarse voltage control in electric power \\ systems. There could be an interaction between load dynamics and OLTC control under certain system \\ operating conditions which may lead to OLTC limit cycle phenomena thereby resulting into long term \\ voltage oscillations in the system. In the recent years, renewable and non-renewable local generation \\ units have been getting interconnected in modern power systems. The existence of OLTC limit cycles in \\ the presence of local generation units has not been addressed thoroughly in the literature. In this paper, \\ the OLTC limit cycle phenomena, which can occur due to interactions among load-voltage dynamics, OLTC \\ controls and the local generation, has been investigated. Also, a strategy is proposed for mitigating the \\ power system oscillations which can be induced by OLTC limit cycles, especially with local generation \\ units in the network. The proposed mitigation strategy including detailed investigations and analyses \\ have been verified for a two-bus system, and successfully tested on a multi-bus system with local \\ generation units using MATLAB.

\section{Disciplines} \\ Engineering | Science and Technology Studies

\section{Publication Details} \\ D. Ranamuka, A. P. Agalgaonkar, K. M. Muttaqi \& M. J. E. Alam, "Mitigating tap-changer limit cycles in \\ modern electricity networks embedded with local generation units," in IEEE Industry Applications Society \\ Annual Meeting, 2014, pp. 1-8.
}




\title{
Mitigating Tap-changer Limit Cycles in Modern Electricity Networks Embedded with Local Generation Units
}

\author{
D. Ranamuka, Student Member, IEEE, A. P. Agalgaonkar, Senior Member, IEEE, K. M. Muttaqi, \\ Senior Member, IEEE, and M. J. E. Alam, Member, IEEE
}

\begin{abstract}
Cascaded on-load tap changers (OLTC) are widely used for coarse voltage control in electric power systems. There could be an interaction between load dynamics and OLTC control under certain system operating conditions which may lead to OLTC limit cycle phenomena thereby resulting into long term voltage oscillations in the system. In the recent years, renewable and non-renewable local generation units have been getting interconnected in modern power systems. The existence of OLTC limit cycles in the presence of local generation units has not been addressed in the literature. In this paper, the OLTC limit cycle phenomena, which can occur due to interactions among loadvoltage dynamics, OLTC controls and the local generation, has been investigated. Also, a strategy is proposed for mitigating the power system oscillations which can be induced by OLTC limit cycles, especially with local generation units in the network. The proposed mitigation strategy including detailed investigations and analyses have been verified for a two-bus system, and successfully tested on a multi-bus system with local generation units using MATLAB.
\end{abstract}

Index Terms--Limit-cycle phenomena; load dynamics; local generation; on-load tap changer; power system oscillations.

\section{INTRODUCTION}

$\mathrm{L}$ IMIT cycles attributed to the operation of on-load tap changers (OLTCs) can occur in electricity networks under certain operating conditions mainly due to the interaction between load dynamics and OLTC controls. Eventually, these limit cycles in case of cascaded tap changers may result into sustained long term oscillations in the power systems. It may also affect the long term voltage stability of the network.

In [1], the existence of OLTC limit cycles is investigated and analyzed. The system load level, degree of reactive power compensation and the load voltage dependency are identified as the key parameters for initiation and avoidance of the OLTC limit cycles. The nature of limit cycles caused by the interaction between transformer tap changer and load dynamics is analyzed in [2]. A linearization of Poincar'e map is used to analyze the local stability in the system under OLTC limit cycles. In [3], voltage oscillations in power systems with

The authors are with the Australian Power Quality and Reliability Center, School of Electrical, Computer and Telecommunications Engineering, University of Wollongong, New South Wales 2522 Australia. (e-mail: dothinkanes@yahoo.co.uk; ashish@uow.edu.au; kashem@uow.edu.au) cascaded multiple OLTC units have been studied, where the focus has been on the limit cycles due to interactions between the tap changer and the load. Also, a control strategy is proposed in [3] to mitigate the OLTC limit cycles. It is based on adjusting the dead-band (DB) of the tap changer, which typically depends on the load characteristics. It has been found that the existing limit cycles will be avoided and a steady state condition is reached given a sufficiently large DB in case of stable load dynamics when tap ratios are fixed. On the other hand, the existing limit cycles will not be removed by increasing DB in case of unstable load dynamics wherein tap ratios are fixed. Moreover, it has been found that adjusting OLTC control parameters such as time delay and/or DB size may not have any effect on the existence of limit cycles under certain system conditions [1]. It may not be possible to avoid limit cycle behavior simply by retuning the OLTC dead-band limit and/or time delay. However, none of the studies in the literature have investigated and analyzed the OLTC limit cycle phenomena in medium voltage (MV) networks with higher penetration of renewable and non-renewable local generation (LG) units. For such networks, OLTC limit cycles can occur frequently due to interactions among load and voltage dynamics, OLTC control and the local generation.

In this paper, OLTC limit cycle phenomena in case of MV networks with higher penetration of LG units is investigated and analyzed thoroughly. The small signal model and describing function method used in [1] for OLTC limit cycle analysis in a two bus system have been extended for analyzing and predicting OLTC limit cycles in different multi bus system topologies with LG units. Also, a strategy based on coordinated VAr support from LG units and capacitor banks (CBs) is proposed to mitigate the OLTC limit cycles in the presence of LG units. It is easily implementable with a typical voltage control scheme. In this paper, MATLAB is used for modeling the sample system and conducting associated simulation studies.

This paper is organized as below. Section II outlines the theoretical background of investigating and analyzing the OLTC limit cycles and Section III elaborates the algorithm of the proposed strategy for mitigating OLTC limit cycles including realistic case study, while the concluding remarks have been made in Section IV of the paper. 


\section{BACKGROUND THEORY}

\section{A. Predicting Existence of OLTC Limit Cycles}

Eigen value analysis is used to predict the existence of OLTC limit cycles, and the results are compared with describing function analysis. For large MV power systems, network reduction methods can be applied to minimize the computational burden [4].

1) Modeling Aspects

The model described by the dead band-ordinary differential equation (DB-ODE) is used for modeling OLTC as given by (01) [5]. It is noted that discrete tap steps are not taken into account in this OLTC model.

$$
\frac{d n}{d t}=\left\{\begin{array}{lll}
(-) \frac{1}{T} \cdot\left(V_{L C}-V_{\text {set }}-D B / 2\right) & \text { if } & \left(V_{L C}-V_{\text {set }}\right)>D B / 2 \\
(-) \frac{1}{T} \cdot\left(V_{L C}-V_{\text {set }}+D B / 2\right) & \text { if } & \left(V_{L C}-V_{\text {set }}\right)<(-) D B / 2 \\
0 & \text { if }\left|V_{L C}-V_{\text {set }}\right|<D B / 2
\end{array}\right.
$$

where $V_{L C}$ denotes regulated voltage at the regulating point, $V_{\text {set }}$ is the voltage set value, $T$ is the controller time delay and $n$ is the transformer tap-ratio.

Accurate modeling of different load characteristics is one of the key requirements of analyzing and predicting OLTC limit cycles. In this paper, the loads are modelled as exponential recovery loads as given by (02) and (03) [4].

$$
\begin{aligned}
& \dot{x}_{p}=\frac{1}{T_{p}}\left(-x_{p}+P_{s}(V)-P_{t}(V)\right), \quad P_{s}(V)=k_{L} \cdot P_{0}(V)^{\alpha_{s}} \\
& P_{t}(V)=k_{L} \cdot P_{0}(V)^{\alpha_{t}}, \quad P_{d}=x_{p}+P_{t}(V) \\
& \dot{x}_{q}=\frac{1}{T_{q}}\left(-x_{q}+Q_{s}(V)-Q_{t}(V)\right), \quad Q_{s}(V)=k_{L} \cdot Q_{0}(V)^{\beta_{s}} \\
& Q_{t}(V)=k_{L} \cdot Q_{0}(V)^{\beta_{t}}, \quad Q_{d}=x_{q}+Q_{t}(V)
\end{aligned}
$$

where, $x$ is an internal state which models the load recovery dynamics. The recovery time constants are $T_{p}$ and $T_{q}$, and $\alpha_{s}$, $\alpha_{t}, \beta_{s}, \beta_{t}$ are the exponents of the voltage. The steady state nodal voltage dependency is denoted using $P_{s}(V)$ and $Q_{s}(V)$, where the transient (instantaneous) nodal voltage dependency is denoted using $P_{t}(V)$ and $Q_{t}(V)$ respectively. The $P_{d}$ and $Q_{d}$ denote actual loads where the rated load values are denoted using $P_{0}$ and $Q_{0}$. The load scale factor is $k_{L}$.

It is assumed that the LG units respond instantaneously to the system changes. The respective power injections of LG units have been incorporated in the power balance equations. The active power response of LG unit is $P_{L G}$ whereas the reactive power response is $Q_{L G}$.

The describing function $(N(A))$ of the dead-band $(D B)$ in the DB-ODE model of the OLTC can be derived as given by (04) [1], [6]. The amplitude of any sinusoidal input is $A$, where periodic OLTC limit cycles are assumed to be approximately sinusoidal. The condition associated with the occurrence of OLTC limit cycle phenomenon is given by (05), where the small signal model of the equivalent system is given by (06).
The system stability under each operation is assessed using the proposed small signal model and the associated eigen value analysis. This is an extended version of the analysis done in [1].

$$
N(A)=\left\{\begin{array}{l}
1-\frac{2}{\pi}\left(\sin ^{-1}\left(\frac{D B}{2 A}\right)+\frac{D B}{2 A} \sqrt{1-\left(\frac{D B}{2 A}\right)^{2}}\right) \text { if } A>D B / 2 \\
0 \text { if } A<D B / 2
\end{array}\right.
$$

Condition for limit cycle phenomenon :

$G(j \omega)=(-)\left\{\frac{1}{N(A)}\right\} \quad \forall G(j \omega)=(-) G_{n}(j \omega) \times G_{c}(j \omega)$

$G_{n}(s) \rightarrow$ transfer function of the model associated with OLTC, which is derived from the state space model given by (06)

$G_{c}(s) \rightarrow$ transfer function of the ODE part of DBODE model

\section{2) Case Study for a Two Bus System}

The two bus system shown in Fig. 1 is used for investigating and analyzing OLTC limit cycle phenomenon under different system operational states.

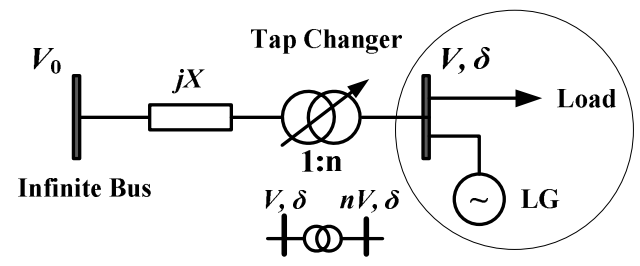

Fig. 1. Two bus system model.

The proposed mathematical model of the power system used for analyzing OLTC limit cycles is given below.

Power system equations including dynamics :

$\dot{x}=f(x, v), \quad g\left(x, v, P_{L G}, Q_{L G}, u\right)=0$

$\delta=$ voltage phasor angle, $V=$ voltage magnitude

State space model of the power system :

$\Delta \dot{x}=\left(\frac{\partial f}{\partial x}\right) \cdot \Delta x+\left(\frac{\partial f}{\partial v}\right) \cdot \Delta v$
$\left(\frac{\partial g}{\partial x}\right) \cdot \Delta x+\left(\frac{\partial g}{\partial v}\right) \cdot \Delta v+\left(\frac{\partial g}{\partial u}\right) \cdot \Delta u=0$

$\Delta \dot{x}=(A) \cdot \Delta x+(B) \cdot \Delta n$

$\Delta v=(C) \cdot \Delta x+(D) \cdot \Delta n$

State matrix : $x=\left[\begin{array}{ll}x_{p} & x_{q}\end{array}\right]^{T}$, Input matrix : $u=[n]$

Output matrix : $v=\left[\begin{array}{ll}V & \delta\end{array}\right]^{T}$

$A=\left(\frac{\partial f}{\partial x}\right)-\left(\frac{\partial f}{\partial v}\right) \cdot\left(\frac{\partial g}{\partial v}\right)^{-1} \cdot\left(\frac{\partial g}{\partial x}\right), \quad C=(-) \cdot\left(\frac{\partial g}{\partial v}\right)^{-1} \cdot\left(\frac{\partial g}{\partial x}\right)$

$B=(-) \cdot\left(\frac{\partial f}{\partial v}\right) \cdot\left(\frac{\partial g}{\partial v}\right)^{-1} \cdot\left(\frac{\partial g}{\partial u}\right), \quad D=(-) \cdot\left(\frac{\partial g}{\partial v}\right)^{-1} \cdot\left(\frac{\partial g}{\partial u}\right)$

The existence of OLTC limit cycles in the presence of LG unit has been tested for different load demand levels, and the 
key results of some example simulations are summarized below. The Nichols plots of both left and right hand side functions are used to solve the equation (05). The sample load and system data, used for simulation purposes, are $P_{0}=106.8$ MW, $Q_{0}=43.2$ MVAr, $X=0.10641 \mathrm{pu}, \alpha_{s}=1, \beta_{s}=0, \alpha_{t}=1$, $\beta_{t}=4$ and $T_{p}=T_{q}=60 \mathrm{~s}$. The tap changer controller time delay $(T)$ is $30 \mathrm{~s}$. The simulated voltage change per tap operation is $0.0010 \mathrm{pu}$. The initial tap position of the OLTC is set at its nominal position for all simulations. The peak load demand is 96.005 MVA, where $k_{L}=1,0.9,0.8,0.7,0.6,0.5,0.4$ and 0.3 . The sending end bus voltage is $1.01 \mathrm{pu}$. Fig. 2 shows the respective Nichols plots for two bus system operation without local generation unit (case-01). According to the Nichols plots (Gki, where $i=1, \ldots, 8$ ), it can be seen that the plots do not intersect the Nichols plot of $-1 / \mathrm{N}$ (A) function for different values of $k_{L}$, which demonstrates that OLTC limit cycles do not exist for the test system without local generation. The plot (GA), shown by the orange color vertical line, represents the Nichols plot of $-1 / \mathrm{N}(\mathrm{A})$ function.

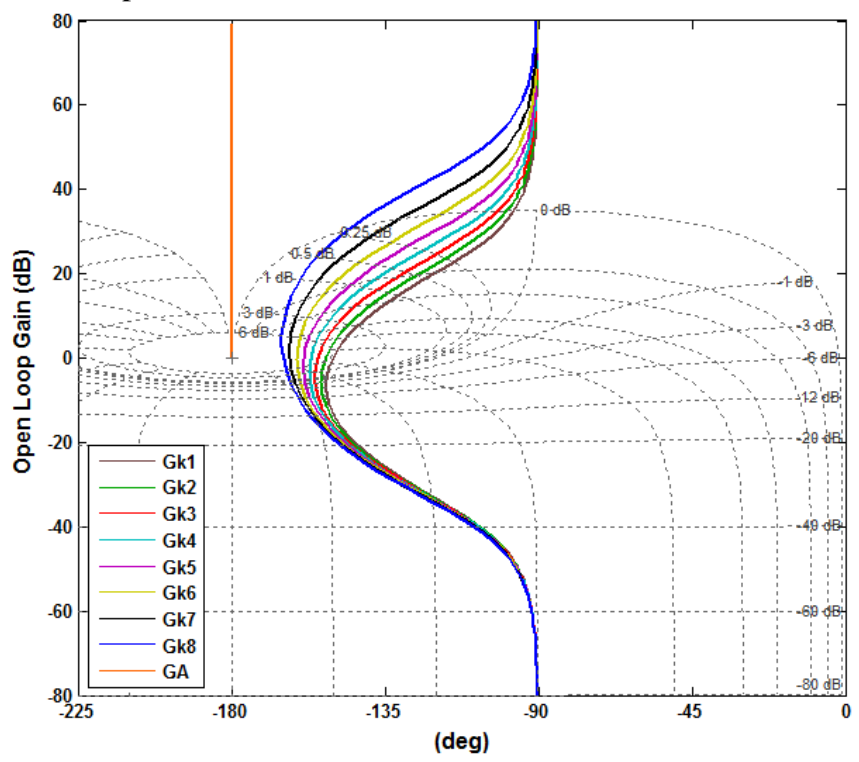

Fig. 2. Nichols plots of $\mathrm{G}(\mathrm{j} \omega)$ function for different values of $k_{L}$ in case of system operations without LG and Nichols plot of $-1 / \mathrm{N}(\mathrm{A})$ (case-01).

According to the investigations, it can be seen that there can be OLTC limit cycles where active power generation level of the LG unit exceeds 26.5 MW and $k_{L}=0.3$ as shown in Fig. 3 (case-02).

The Fig. 4 illustrates an example OLTC limit cycle phenomenon in real time for the predicted case- 02 shown in Fig. 3. This is obtained by solving the first order differential equations of $x$-states which models the load recovery dynamics. In this case, the power output of the LG unit is assumed to be constant, where mechanical time delay of OLTC is assumed to be $6 \mathrm{~s}$. The time domain simulation studies highlight the suitability of describing function method for predicting OLTC limit cycles in electric power systems.

The Fig. 5 shows that the OLTC limit cycles may recur frequently if active power generation level of the LG unit exceeds 87.5 MW (case-03).

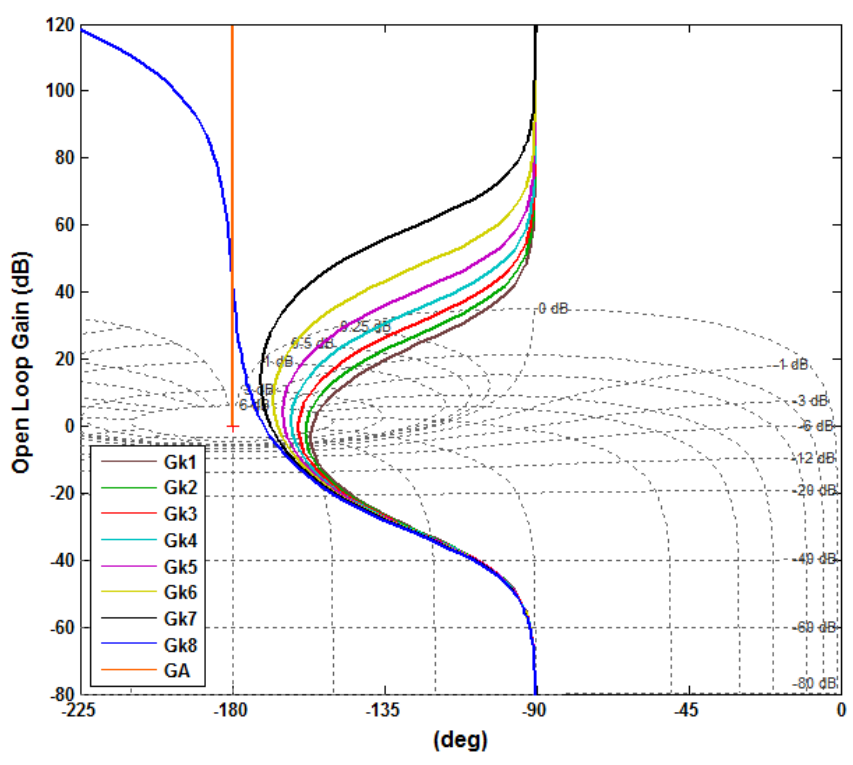

Fig. 3. Nichols plots of $\mathrm{G}(\mathrm{j} \omega)$ function for different values of $k_{L}$ in case of system operations with $\mathrm{P}_{\mathrm{LG}}=26.5 \mathrm{MW}$ (case-02).

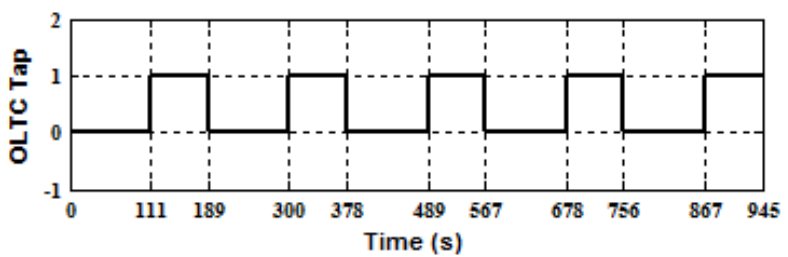

(a)

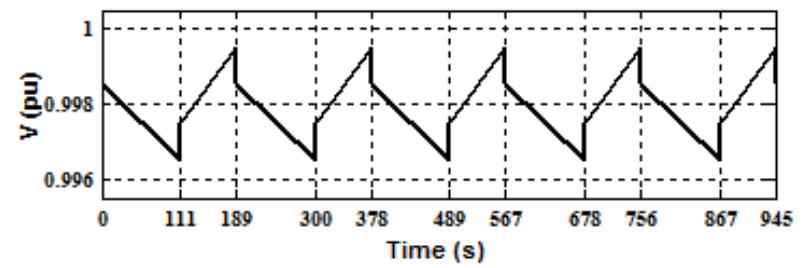

(b)

Fig. 4. Simulated (a) OLTC tap operations and (b) resultant voltage oscillations, which can be occurred due to OLTC limit cycle phenomenon predicted in case- 02 .

Importantly, it is very clear that the recurrence of OLTC limit cycles in the presence of a LG unit under any practical system operation can be high, as predicted, compared to the system operation without a LG unit, due to variation and intermittency in the power output of LG unit and associated change in dynamics. In case of multiple OLTC units, hunting among OLTCs is another issue. In such a situation, predicted limit cycles for a particular OLTC may not be of sustained nature and it may be diminished after the next tap operation in the system. Also, the limit cycles may sustain for a longer time due to the variation in the power output by a LG unit and the associated change in system dynamics, especially when the load factor, $k_{L}$ do not vary (flat load profiles) or has slow ramp variation. Therefore, an implementation strategy for mitigating OLTC limit cycles in the presence of LG units may be essential for networks with high penetration of local generation. 


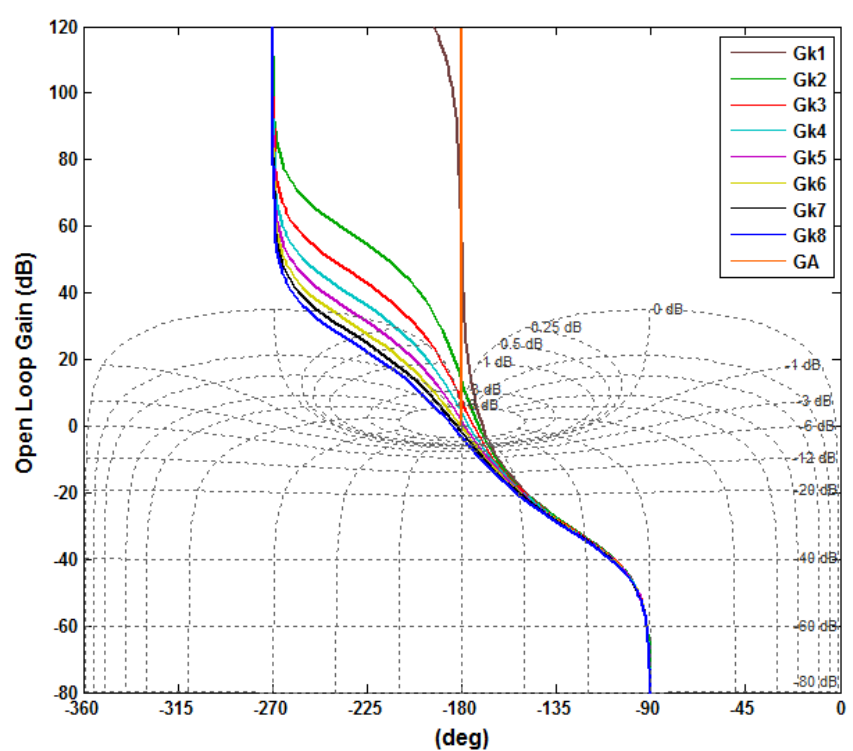

Fig. 5. Nichols plots of $\mathrm{G}(\mathrm{j} \omega)$ function for different values of $k_{L}$ in case of system operations with $\mathrm{P}_{\mathrm{LG}}=87.5 \mathrm{MW}$ (case-03).

The reactive power support of 13.5 MVAr by the LG unit in Fig. 1 can prevent the system from an oscillatory response, attributed to OLTC limit cycles, which can occur when the real power output of the LG unit is $26.5 \mathrm{MW}$ and $k_{L}=0.3$ as shown in Fig. 6 (case-04). It is an indicative of the fact that OLTC limit cycles may be mitigated by implementing a coordinated $\mathrm{VAr}$ management scheme in the system, comprising of Volt/VAr support by the LG unit.

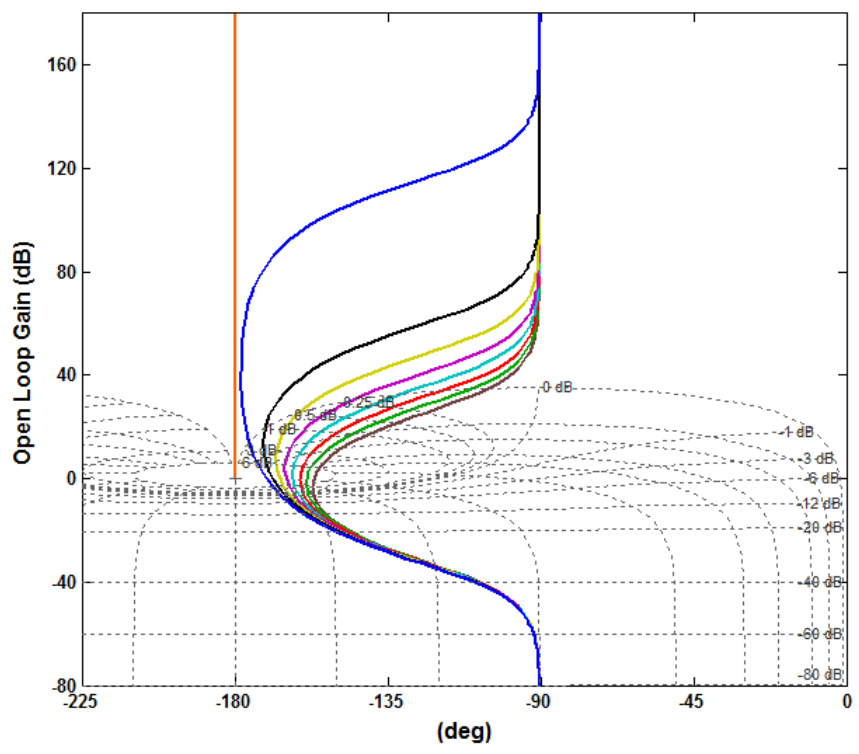

Fig. 6. Nichols plots of $\mathrm{G}(\mathrm{j} \omega)$ function for different values of $k_{L}$ in case of system operations with $P_{L G}=26.5 \mathrm{MW}$ and $\mathrm{Q}_{\mathrm{LG}}=13.5 \mathrm{MVAr}$ (case-04).

When the LG unit absorbs reactive power (case-05), the intersection point of the associated Nichols plots i.e., Gki (where $k_{L}=0.3$ ) is shifted downward as shown in Fig. 7 along the GA curve to a lower open loop gain compared to the case02 . It means that the absorption of reactive power by the LG unit affects $G(j \omega)$ function (Gki) as a gain factor.
Consequently, the amplitude of limit cycles is changed, but not the frequency. Moreover, shifting the curve below the point $\left(0 \mathrm{~dB},-180^{\circ}\right)$ can remove a limit cycle, but it may lead to instability of the closed loop system. In summary, it is clear that there would be a certain LG penetration level which can create OLTC limit cycles, and also which can mitigate OLTC limit cycles for each operational state of the system.

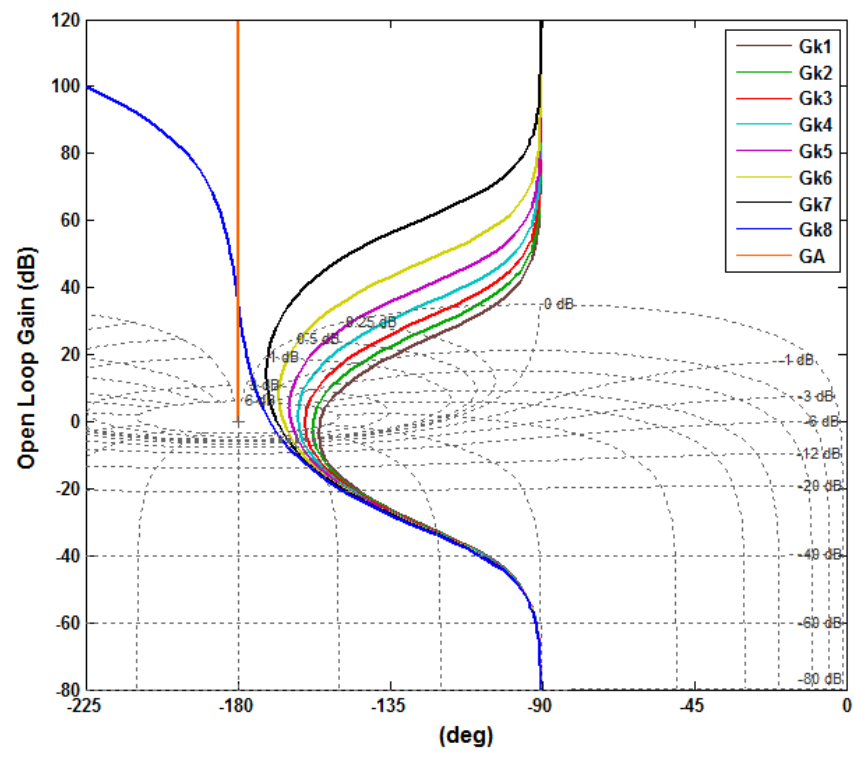

Fig. 5. Nichols plots of $\mathrm{G}(\mathrm{j} \omega)$ function for different values of $k_{L}$ in case of system operations with $\mathrm{P}_{\mathrm{LG}}=26.5 \mathrm{MW}$ and $\mathrm{Q}_{\mathrm{LG}}=-9.6 \mathrm{MVAr}$ (case-05).

Finally, the eigen values of overall system (i.e. combined ODE part of the OLTC model and power system model) state matrix for above different operations (case-01 to case-05) are derived and shown in Table I. The unstable scenarios with OLTC limit cycles, where at least one of the eigen values has a positive real part are highlighted. The results of the eigen value analysis are very much in agreement with the results obtained using describing function method, which has been implemented for predicting the existence of OLTC limit cycles. Moreover, a modal analysis can be done using the proposed small signal model in order to identify the oscillatory modes referred to OLTC limit cycle instability; which is out of the scope of this paper.

\section{B. Case Study for a Multi Bus System}

In this case study, OLTC limit cycle phenomenon is investigated and analyzed for a multi-bus system, as shown in Fig. 8, for different system operational states, and one of the simulated cases is presented in this paper. The describing function analysis and eigen value analysis are carried out, and compared for multi bus systems in order to further test the applicability of the mathematical model derived under Section II- $A$ for predicting the OLTC limit cycles. The multi-bus system of Fig. 8 is derived from [7] and modified by adding load dynamics, control data and the line data (i.e., $R_{5}=$ $0.00192 \mathrm{pu}$ and $X_{5}=0.04256 \mathrm{pu}$ ) related to connecting the LG unit. 
TABLE I

RESUlts of EIGEN VALUE ANALYSIS FOR THE Two BUS SySTEM OPERATION

\begin{tabular}{|c|c|c|c|c|}
\hline \multirow{2}{*}{$\mathrm{k}_{\mathrm{L}}$} & 1.0 & 0.9 & 0.8 & 0.7 \\
\hline \multirow{2}{*}{ Case-01 } & -0.0010 & -0.0009 & -0.0008 & -0.0007 \\
& -0.0167 & -0.0167 & -0.0167 & -0.0167 \\
\hline \multirow{2}{*}{ Case-02 } & -0.0007 & -0.0006 & -0.0005 & -0.0004 \\
& -0.0167 & -0.0167 & -0.0167 & -0.0167 \\
\hline \multirow{2}{*}{ Case-03 } & +0.0000 & +0.0001 & +0.0002 & +0.0003 \\
& -0.0167 & -0.0167 & -0.0167 & -0.0167 \\
\hline \multirow{2}{*}{ Case-04 } & -0.0007 & -0.0006 & -0.0005 & -0.0004 \\
& -0.0167 & -0.0167 & -0.0167 & -0.0167 \\
\hline \multirow{2}{*}{ Case-05 } & -0.0007 & -0.0006 & -0.0005 & -0.0004 \\
& -0.0167 & -0.0167 & -0.0167 & -0.0167 \\
\hline \multirow{2}{*}{$\mathrm{k}_{\mathrm{L}}$} & 0.6 & 0.5 & 0.4 & 0.3 \\
\hline \multirow{2}{*}{ Case-01 } & -0.0006 & -0.0005 & -0.0004 & -0.0003 \\
& -0.0167 & -0.0167 & -0.0167 & -0.0167 \\
\hline \multirow{2}{*}{ Case-02 } & -0.0003 & -0.0002 & -0.0001 & +0.00000 \\
& -0.0167 & -0.0167 & -0.0167 & -0.0167 \\
\hline \multirow{2}{*}{ Case-03 } & +0.0004 & +0.0005 & +0.0006 & +0.0007 \\
& -0.0167 & -0.0167 & -0.0167 & -0.0167 \\
\hline \multirow{2}{*}{ Case-04 } & -0.0003 & -0.0002 & -0.0001 & -0.0000 \\
& -0.0167 & -0.0167 & -0.0167 & -0.0167 \\
\hline \multirow{2}{*}{ Case-05 } & -0.0003 & -0.0002 & -0.0001 & +0.00001 \\
& -0.0167 & -0.0167 & -0.0167 & -0.0167 \\
\hline
\end{tabular}

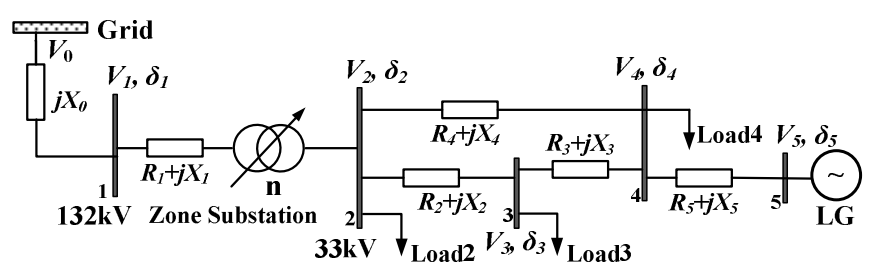

Fig. 8. Multi bus system model.

The small signal model of the multi bus power system is given by (07)

System equations including dynamics :

$\dot{x}=f(x, v), \quad g\left(x, v, P_{L G}, Q_{L G}, u\right)=0$

State space model :

$\Delta \dot{x}=\left(\frac{\partial f}{\partial x}\right) \cdot \Delta x+\left(\frac{\partial f}{\partial v}\right) \cdot \Delta v$

$\left(\frac{\partial g}{\partial x}\right) \cdot \Delta x+\left(\frac{\partial g}{\partial v}\right) \cdot \Delta v+\left(\frac{\partial g}{\partial u}\right) \cdot \Delta u=0$

$\Delta \dot{x}=\left(A_{1}\right) \cdot \Delta x+\left(B_{1}\right) \cdot \Delta n$

$\Delta v=\left(C_{1}\right) \cdot \Delta x+\left(D_{1}\right) \cdot \Delta n$

State matrix : $x=\left[\begin{array}{llllll}x_{p 2} & x_{q 2} & x_{p 3} & x_{q 3} & x_{p 4} & x_{q 4}\end{array}\right]^{T}$

Input matrix : $u=[n]$

Output matrix : $v=\left[\begin{array}{llllll}V_{2} & V_{3} & V_{4} & \delta_{2} & \delta_{3} & \delta_{4}\end{array}\right]^{T}$

$A_{1}=\left(\frac{\partial f}{\partial x}\right)-\left(\frac{\partial f}{\partial v}\right) \cdot\left(\frac{\partial g}{\partial v}\right)^{-1} \cdot\left(\frac{\partial g}{\partial x}\right), \quad C_{1}=(-) \cdot\left(\frac{\partial g}{\partial v}\right)^{-1} \cdot\left(\frac{\partial g}{\partial x}\right)$

$B_{1}=(-) \cdot\left(\frac{\partial f}{\partial v}\right) \cdot\left(\frac{\partial g}{\partial v}\right)^{-1} \cdot\left(\frac{\partial g}{\partial u}\right), \quad D_{1}=(-) \cdot\left(\frac{\partial g}{\partial v}\right)^{-1} \cdot\left(\frac{\partial g}{\partial u}\right)$

For the tap changer model, input is the transformer secondary bus voltage magnitude, $V_{2}$ whereas output is the tap ratio, $n$. The OLTC limit cycles can be predicted as shown in Fig. 9, where the simulated load and system data are as below: total $P_{0}=94.0 \mathrm{MW}$, total $Q_{0}=21.0 \mathrm{MVAr}, \alpha_{s}=\beta_{s}=1, \alpha_{t}=2$, $\beta_{t}=4, T_{p}=120 \mathrm{~s}, T_{q}=60 \mathrm{~s}$ and OLTC controller time delay, $T$ $=30 \mathrm{~s}$. The total active and reactive power outputs by the LG unit are 34.6 MW and 5.3 MVAr, respectively. Initial tap position of OLTC is ' 1 ' in the direction of increasing voltage, where taps are incorporated in the primary winding of the substation transformer. The total peak load demand is around 90.0 MVA, where $k_{L}=0.85$ and the grid voltage is $1.0 \mathrm{pu}$.

The eigen values derived using overall system state matrix with the VAr support by the LG unit are shown in Table II.

TABLE II

Results of EIGEN VALUe ANALYSIS FOR THE MULTi BUS SySTEM

\begin{tabular}{|c|c|c|c|c|c|}
\hline \multicolumn{6}{|c|}{ OPERATION WITH VAR SUPPORT OF THE LG UNIT } \\
\hline $\begin{array}{c}0.0010+ \\
0.0008 \mathrm{i}\end{array}$ & $\begin{array}{c}0.0010- \\
0.0008 \mathrm{i}\end{array}$ & -0.0186 & -0.0174 & -0.0078 & -0.0081 \\
\hline
\end{tabular}

They are indicative of the fact that OLTC limit cycles can also exist with the LG unit operating in voltage control mode, especially when the control action of the LG unit has not been coordinated with the operation of other voltage regulating devices. Also, this simulation shows the applicability and suitability of proposed eigen value analysis for predicting OLTC limit cycles in multi bus systems. Therefore, the proposed strategy in section-III for mitigating OLTC limit cycles is mainly based on the proposed mathematical model given by (06) and the associated eigen value analysis which can be used for predicting OLTC limit cycles in electricity networks with multiple LG units.

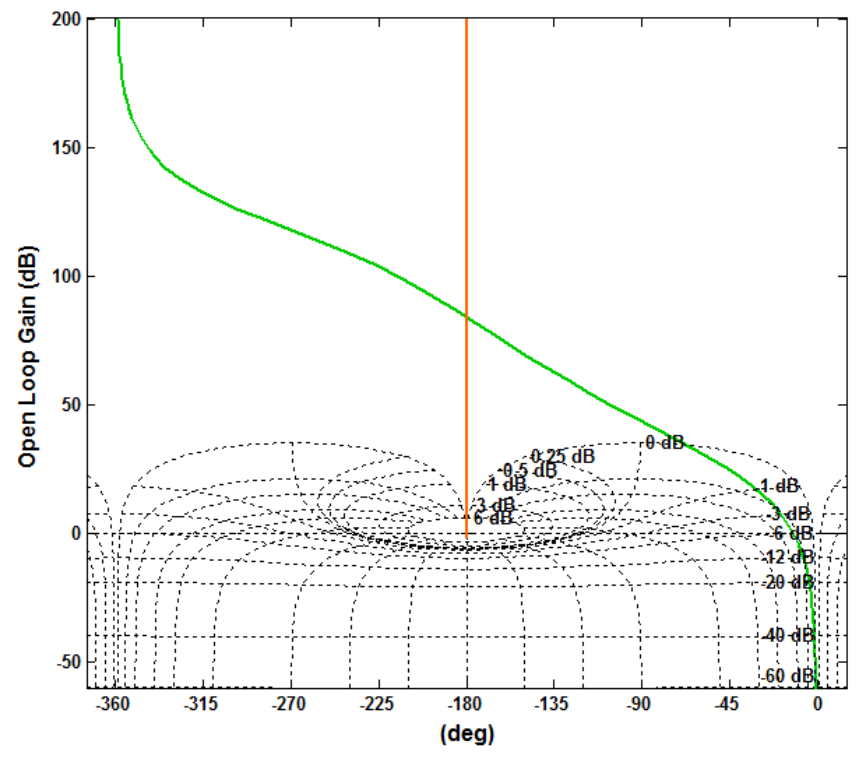

Fig. 9. Nichols plots of $\mathrm{G}(\mathrm{j} \omega)$ for an existence of OLTC limit cycles in the multi bus system when $k_{L}=0.85, \mathrm{P}_{\mathrm{LG}}=34.6 \mathrm{MW}$ and $\mathrm{Q}_{\mathrm{LG}}=5.3 \mathrm{MVAr}$.

\section{Proposed Control Strategy for Mitigating OLTC LIMIT CYCLES IN PRESENCE OF LG UNITS}

From power system control perspective, the systemoperating conditions are normally classified into five states such as normal, alert, emergency, extreme emergency and restorative [8]. In the proposed strategy for mitigating OLTC 
limit cycles, the control transition is between normal and the alert states. The system enters the alert-state form the normalstate, if existence of OLTC limit cycles is predicted. Then, the preventive control action based on the proposed control strategy is activated to mitigate the OLTC limit cycles while controlling the system voltage. Since the objectives of normal state voltage control can be different and incorporating those objectives with mitigating OLTC limit cycles may not always be effective, this transition based voltage control is used. Also, it is proposed to embed the proposed control module in a centralized voltage control scheme as additional hardware and software for updated operation, thereby ensuring effective voltage control.

\section{A. Proposed Strategy}

This is mainly based on coordinated VAr support from LG units and $\mathrm{CBs}$ for avoiding the conditions which have to be satisfied for the existence of OLTC limit cycles. From (06) and for particular operation of OLTCs and CBs, it can be seen that the matrix- $A$ is a function of nodal voltage magnitudes and phase angles as given by (08).

$$
\begin{aligned}
& A=\left(\frac{\partial f}{\partial x}\right)-\left(\frac{\partial f}{\partial v}\right) \cdot\left(\frac{\partial g}{\partial v}\right)^{-1} \cdot\left(\frac{\partial g}{\partial x}\right) \\
& \left(\frac{\partial f}{\partial v}\right)=f(V), \quad\left(\frac{\partial g}{\partial v}\right)=f(V, \delta) \rightarrow A=f(V, \delta)
\end{aligned}
$$

Hence, by means of voltage control through coordinated VAr management in the system, a stable system operation without system oscillations, typically induced by OLTC limit cycles, can also be achieved. Accordingly, the proposed mitigating strategy is designed. The step-by-step algorithm of the proposed strategy is outlined below.

Step-01: From the on-line measurements and information sent by distribution management system (DMS), the control module is executed.

Step-02: For the current state of the system, the overall system state matrix is updated and the respective eigen values are derived.

Step-03: If all the eigen values have negative real part, the normal state voltage control module is enacted.

Step-04: If at least one eigen value has a positive real part, the alert state voltage control module is enacted.

Step-05: The sensitivity matrix, $S_{M}$, given by (09) is derived. The sensitivity values of VAr supports by the LG unit and the CB are $S_{M Q L G}$ and $S_{M Q C B}$, respectively, where $\Delta V$ is voltage deviation for small change of the LG unit's reactive power, $\Delta Q_{L G}$ and CB's reactive power, $\Delta Q_{C B}$.

$$
\Delta V=\left[\begin{array}{l:l}
S_{M Q L G} & S_{M Q C B}
\end{array}\right] \cdot\left[\begin{array}{c}
\Delta Q_{L G} \\
\hdashline \Delta \bar{Q}_{C B}
\end{array}\right], \quad S_{M}=\left[\begin{array}{lll}
S_{M Q L G} & S_{M Q C B}
\end{array}\right]
$$

Step-06: The order of VAr support devices (i.e. LG units and CBs) which are going to be utilized for coordinated VAr support is determined using the associated sensitivity values derived in Step-05, capability of devices for supporting the system voltage and the amount of VAr support. The generalized sequence in terms of time delays, $T$ is given by (10).

$$
\begin{aligned}
& T_{L G \text {-larg er }}<\ldots<T_{L G \text {-smaller }}<T_{C B \text {-larg er }}<\ldots<T_{C B \text {-smaller }} \\
& <\text { OLTC }_{\text {upstream }}<\ldots<O L T C_{\text {downstream }}
\end{aligned}
$$

Step-07: The new VAr reference values for selected VAr support devices (i.e. LG units and CBs) are identified subject to system constraints and capability limits of the LG units and $\mathrm{CBs}$, where objective is to ensure stable system operation without OLTC limit cycles and maintain the system voltage within stipulated limits.

Step-08: The updated VAr reference values are assigned for local controllers of LG units and CBs.

Step-09: The OLTC local controllers are enabled.

Step-10: For the subsequent instances of time (i.e., $t=t+1$ ), repeat the procedure from Step-01.

Flow chart of the proposed voltage control algorithm prior to enacting OLTC tap operations is shown in Fig. 10.

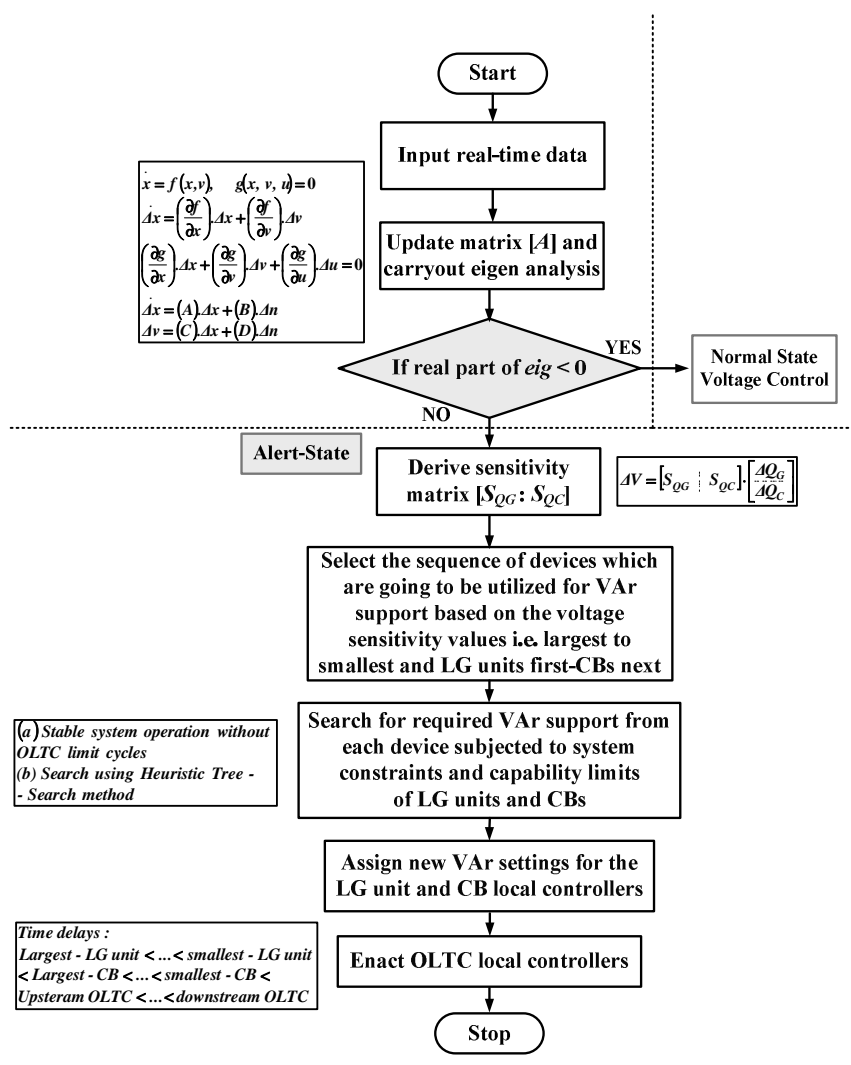

Fig. 10. Flow chart of the proposed voltage control algorithm with capability of mitigating OLTC limit cycles.

The voltage control strategy proposed in [9] can be used for normal state voltage control in conjunction with the proposed strategy of mitigating OLTC limit cycles. It is an online voltage control strategy which is designed and tested for correcting the system voltage with control-coordination ascertaining voltage support by LG units in the system. It also ensures prioritized operation of the voltage support operation of LG units and the voltage regulating devices, and aids in 
blocking simultaneous operations, thereby minimizing the total tap operations. However, even under this normal state voltage control only, there could be a possibility of recurrence of OLTC limit cycles.

Design of the proposed control module contains the embedded mathematical model of the power system, model of the proposed control logic, search engine and the decision making control layer for enacting the VAr controllers of LG units, CBs and the tap operations of OLTCs. The search engine based on the proposed control algorithm, as detailed in the flow chart in Fig. 8, is adopted in order to determine the control parameters of LG units and CBs.

The practical implementation strategy for proposed control is outlined in Fig. 11. The proposed control modules are embedded in a grid centered DMS for on-line voltage control in the given MV test system. The control panels (CPs) of LG units and voltage regulating devices are proposed to be equipped with supervisory control and data acquisition (SCADA) facilities.

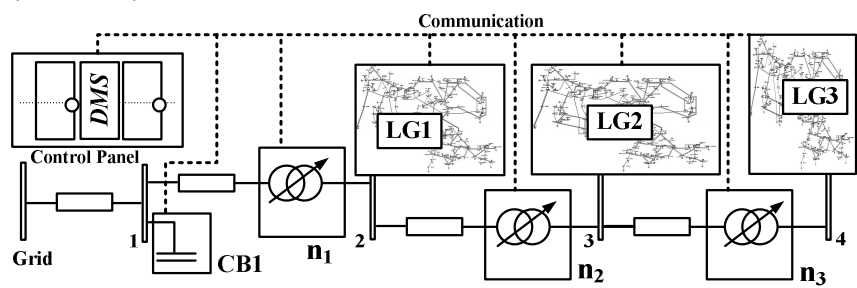

Fig. 11. Topology of the on-line implementation of proposed control strategy (with reference to the test system depicted in Fig. 12).

\section{B. Test Case Study}

\section{1) Control Model}

In this case study, a MV test system with cascaded OLTCs is considered. This sample test system is operated with 3 OLTCs, LG units and a CB for Volt/VAr correction, and its topological model is given by Fig. 12. In this test system, OLTC limit cycles can be induced not only due to interaction among load dynamics, OLTC control and the power generated by LG units, but also due to interaction of $\mathrm{CB}$. This $\mathrm{CB}$ is modeled using its susceptance value, $B$.

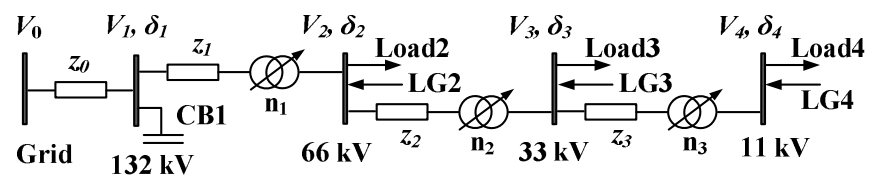

Fig. 12. Model of the test power system used for control model.

The small signal model of the test power system model incorporated in the control model is given by (11).

System equations including dynamics :

$\dot{x}=f(x, v), \quad g\left(x, v, P_{L G}, Q_{L G}, B, u\right)=0, \quad Q_{C B}=B \cdot V_{1}^{2}$

State space model :

$\Delta \dot{x}=\left(\frac{\partial f}{\partial x}\right) \cdot \Delta x+\left(\frac{\partial f}{\partial v}\right) \cdot \Delta v$ $\left(\frac{\partial g}{\partial x}\right) \cdot \Delta x+\left(\frac{\partial g}{\partial v}\right) \cdot \Delta v+\left(\frac{\partial g}{\partial u}\right) \cdot \Delta u=0$

$\Delta \dot{x}=\left(A_{2}\right) \cdot \Delta x+\left(B_{2}\right) \cdot \Delta u$

$\Delta v=\left(C_{2}\right) \cdot \Delta x+\left(D_{2}\right) \cdot \Delta u$

State matrix : $x=\left[\begin{array}{llllll}x_{p 2} & x_{q 2} & x_{p 3} & x_{q 3} & x_{p 4} & x_{q 4}\end{array}\right]^{T}$

Input matrix : $u=\left[\begin{array}{lll}n_{1} & n_{2} & n_{3}\end{array}\right]^{T}$

Output matrix : $v=\left[\begin{array}{llllll}V_{2} & V_{3} & V_{4} & \delta_{2} & \delta_{3} & \delta_{4}\end{array}\right]^{T}$

$A_{2}=\left(\frac{\partial f}{\partial x}\right)-\left(\frac{\partial f}{\partial v}\right) \cdot\left(\frac{\partial g}{\partial v}\right)^{-1} \cdot\left(\frac{\partial g}{\partial x}\right), \quad C_{2}=(-) \cdot\left(\frac{\partial g}{\partial v}\right)^{-1} \cdot\left(\frac{\partial g}{\partial x}\right)$

$B_{2}=(-) \cdot\left(\frac{\partial f}{\partial v}\right) \cdot\left(\frac{\partial g}{\partial v}\right)^{-1} \cdot\left(\frac{\partial g}{\partial u}\right), \quad D_{2}=(-) \cdot\left(\frac{\partial g}{\partial v}\right)^{-1} \cdot\left(\frac{\partial g}{\partial u}\right)$

\section{2) Test Results}

In the test case study, different system operational states are simulated and one of the cases is presented. In this state, a possibility of limit cycles in OLTC $\left(n_{1}\right)$ and OLTC $\left(n_{2}\right)$ is predicted according to Step-01 to Step-03 of the proposed control algorithm detailed earlier in Section III-A. The describing function analysis shown in Fig. 13 confirms the results given by proposed eigen value analysis which are shown in Table III. As discussed in Section II- $A$, the predicted OLTC limit cycles may or may not be sustained. However, enacting a mitigating strategy would be essential in a network, such as above test system, with a significant penetration of local generation, because there is a higher possibility for sustaining the predicted OLTC limit cycles due to intermittency in power outputs of the LG units and associated change in dynamics.

The simulated load scale factors for load 2, 3, and 4 are $k_{L 2}$ $=0.8, k_{L 3}=0.9$, and $k_{L 4}=0.9$, where total peak load demands are $80 \mathrm{MVA}, 12.800 \mathrm{MVA}$ and $3.128 \mathrm{MVA}$, and total $P_{02}=$ $86.4 \mathrm{MW}, Q_{02}=41.8454 \mathrm{MVAr}, P_{03}=13.824 \mathrm{MW}, Q_{03}=$ $6.6953 \mathrm{MVAr}, P_{04}=3.456 \mathrm{MW}$ and $Q_{04}=1.6738 \mathrm{MVAr}$, respectively. The rating of the $\mathrm{CB}$ is $40 \mathrm{MVAr}$ and simulated VAr support is 20 MVAr. The simulated initial tap positions of OLTC $\left(n_{1}\right),\left(n_{2}\right)$ and $\left(n_{3}\right)$ are 2, 4 and 4 respectively in the direction of increasing voltage, where the controller time delays are $30 \mathrm{~s}, 45 \mathrm{~s}$ and $60 \mathrm{~s}$ respectively. The simulated active and reactive power generations of LG1, LG2 and LG3 units are (33.000 MW, 9.300 MVAr), (6.500 MW, 0 MVAr) and (1.600 MW, 0 MVAr), respectively. The simulated load parameters of load 2, 3 and 4 are $\left(\alpha_{s 2}=1.5, \beta_{s 2}=4.5, \alpha_{t 2}=8\right.$, $\left.\beta_{t 2}=3, T_{p 2}=174 \mathrm{~s}, T_{q 2}=84 \mathrm{~s}\right),\left(\alpha_{s 3}=2.5, \beta_{s 3}=5.5, \alpha_{t 3}=4, \beta_{t 3}\right.$ $\left.=1.5, T_{p 3}=201 \mathrm{~s}, T_{q 3}=48 \mathrm{~s}\right)$ and $\left(\alpha_{s 4}=1, \beta_{s 4}=3.5, \alpha_{t 4}=6, \beta_{t 4}\right.$ $\left.=2, T_{p 4}=121 \mathrm{~s}, T_{q 4}=64 \mathrm{~s}\right)$, respectively. The line data as shown in Fig. 10 are $z_{0}=(0.0129+\mathrm{j} 0.0550) \mathrm{pu}, z_{1}=(0.0011$ $+\mathrm{j} 0.0950) \mathrm{pu}, z_{2}=(0.1510+\mathrm{j} 0.6721) \mathrm{pu}$, and $z_{3}=(0.1989+$ $\mathrm{j} 2.6565) \mathrm{pu}$, respectively. The simulated grid voltage is 1.01 $\mathrm{pu}$. 


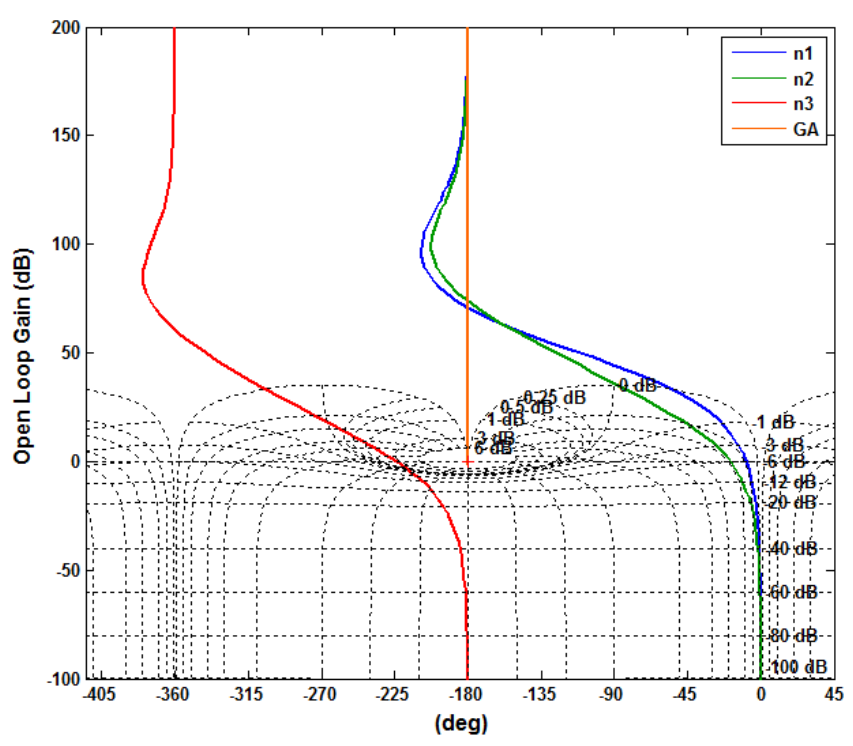

Fig. 13. Nichols plots of $\mathrm{G}(\mathrm{j} \omega)$ for existence of limit cycles in OLTC1 and OLTC 2 under simulated test conditions without proposed mitigation strategy.

TABLE III

RESUlts OF EIGEN VALUE ANALYSIS FOR THE TEST SySTEM OPERATION WITHOUT PROPOSED MITIGATION STRATEGY

\begin{tabular}{|l|l|l|l|l|l|}
\hline+0.0140 & -0.0227 & -0.0139 & -0.0086 & -0.0010 & -0.0036 \\
\hline
\end{tabular}

The voltages at buses 1, 2, 3, and 4 are $1.002 \mathrm{pu}, 0.990 \mathrm{pu}$, $0.964 \mathrm{pu}$ and $0.951 \mathrm{pu}$, respectively. Subsequently (as in the Step-04 of the proposed algorithm), voltage control is moved to the alert state. According to Step-05, the sensitivity matrix, $S_{M}$ is derived. Next, the sequence of VAr support devices (i.e. LG units and $\mathrm{CB}$ ) which are going to be utilized for coordinated VAr support is determined as in Step-06. According to Step-07, the new VAr reference values for selected VAr support devices are derived and they are 10.200 MVAr, 1.900 MVAr, 0.300 MVAr and 25 MVAr, respectively in the order of LG2, LG3, LG4 and CB. In this case, the voltages at buses 1, 2, 3, and 4 are $1.007 \mathrm{pu}, 0.998 \mathrm{pu}, 0.988$ $\mathrm{pu}$ and $0.985 \mathrm{pu}$, respectively. The associated describing function analysis and eigen values are shown in Fig. 14 and Table IV, respectively. Finally, the updated VAr reference values are assigned for the local controllers of LG units and $\mathrm{CB}$, and the OLTC local controllers are enabled according to Step-08 and Step-09, respectively of the proposed algorithm.

TABLE IV

RESUlts of EIGEN VAlue ANALYSIS FOR THE TEST SyStem OPERATION With \begin{tabular}{|c|c|c|c|c|c|}
\hline \multicolumn{6}{|c|}{ PROPOSED MitiGATION STRATEGY } \\
\hline-0.0097 & -0.0200 & $-0.0094+$ & $-0.0094-$ & -0.0023 & -0.0037 \\
\hline
\end{tabular}

According to the above analyses and simulation studies, it is very clear that the proposed methodology of mitigating OLTC limit cycles is applicable to any network, after carefully simplifying and modeling the system and the control logic, compatible to implement the proposed algorithm.

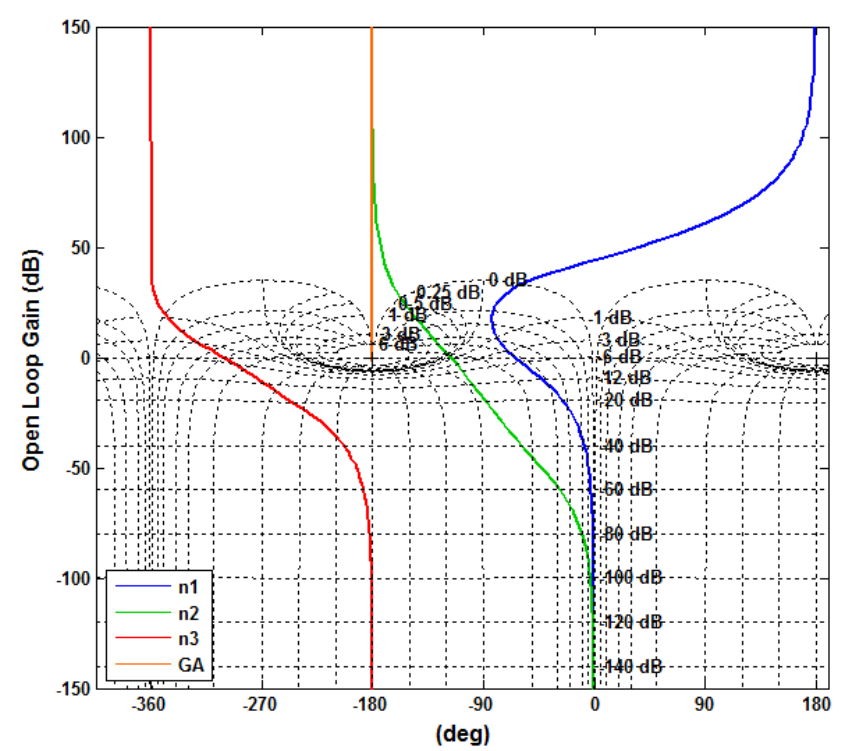

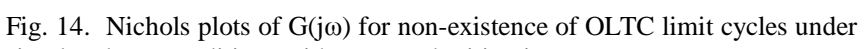
simulated test conditions with proposed mitigation strategy.

\section{CONCLUSION}

In summary, contributions of this paper are (a) development of small signal model and application of describing function method for analyzing OLTC limit cycles in multi bus systems with LG units, (b) investigation and analysis of OLTC limit cycle phenomenon in presence of LG units with voltage control capabilities, and (c) proposal detailing a new strategy for mitigating OLTC limit cycles in presence of LG units, which is designed for alert state voltage control in conjunction with normal state voltage control. It is found that the OLTC limit cycles can frequently be occurred and sustained in electricity networks with LG units due to interactions among load dynamics, OLTC control and the time varying nature of power being generated by LG units, compared to system operations without LG units. Also, it is found that the OLTC limit cycles can be mitigated by coordinated VAr management in the system including prioritized VAr support by the LG units. On-line application of the proposed control strategy will effectively mitigate the sustained oscillations attributed to OLTC limit cycles in networks with LG units.

\section{REFERENCES}

[1] M. Larsson, D. H. Popovic, and D. J. Hill, "Limit Cycles in Power Systems due to OLTC Deadbands and Load-Voltage Dynamics," Electric Power Systems Research, vol. 47, no. 3, pp. 181-188, Nov. 1998.

[2] V. Donde, and I. A. Hiskens, "Analysis of Limit Cycle Stability in A TapChanging Transformer," in Proc. 2002 IEEE Circuits and Systems Conf., pp. 693-696.

[3] Q. Wu, D. H. Popovic, and D. J. Hill, “Avoiding Sustained Oscillations in Power Systems with Tap Changing Transformers," International Journal of Electrical Power \& Energy Systems, vol. 22, no. 8, pp. 597605, Nov. 2000.

[4] R. Lind, and D. Karlsson, "Distribution System Modelling for Voltage Stability Studies," IEEE Trans. Power Systems, vol. 11, no. 4, pp. 16771682, Nov. 1996.

[5] J. Medanic, M. Ilic-Spong, and J. Christensen, "Discrete Models of Slow Voltage Dynamics for Under Load Tap-Changing Transformer 
Coordination," IEEE Trans. Power Systems, vol. 2, no. 4, pp. 873-880, Nov. 1987.

[6] J. C. Hsu, and A. U. Meyer, Modern Control Principles and Applications, vol. 1, New York: McGraw-Hill, 1968.

[7] N. Jenkins, R. Allan, P. Crossley, D. Kirschen and G. Strbac, Embedded Generation, vol. 1. London: Cambridge University Press, 2000, pp. 4963.

[8] P. Kundur, Power System Stability and Control, vol. 1. New York: McGraw-Hill, 1994.

[9] D. Ranamuka, A. P. Agalgaonkar, and K. M. Muttaqi, "On-line Voltage Control in Distribution Systems with Multiple Voltage Regulating Devices," IEEE Trans. Sustainable Energy, vol. 5, no. 2, pp. 617-628, Apr. 2014. 